九州大学学術情報リポジトリ

Kyushu University Institutional Repository

Estimate of the number of defectives when inspectors may make miss checks

Kono, Kazumasa

General Education Department, Kyushu University

Uranishi, Hisao

General Education Department, Kyushu University

https://doi.org/10.5109/13001

出版情報: 統計数理研究. 10 (1/2)，pp.57-68，1961-10. Research Association of Statistical Sciences

バージョン：

権利関係 : 


\title{
ESTIMATE OF THE NUMBER OF DEFECTIVES WHEN INSPECTORS MAY MAKE MISS CHECKS
}

\author{
By \\ K. KôNO and H. URANISI \\ General Education Department, Kyushu University \\ (Received March 30, 1961)
}

1. Introduction and Summary Our problem is not that of sampling inspection. It concerns with a check inspection for acceptance of lots, and surveillance of parts of lines or of those of machines. An inspector examines whether each item is defective or not, or whether each part of line or of machine is abnormal or not. He often overlooks the defectives or the abnormals, while he never makes mistake for the acceptables, or the normal parts. In such circumstances, we are often faced with the following questions. What is the number $N$ of defectives in a lot? How large is the ratio $p$ of correct checks to defectives or abnormals by an inspector? If $p$ is known, then it will be easy to estimate $N$ from the number $y$ of defectives pointed out by the inspector. One of unbiased estimates for $N$ is $y / p$. If $N$ is known, then we can estimate $p$ by $y / N$. Let $p$ and $N$ be unknown. Then, what should we take for the estimates of $N$ and of $p$ ? Our purpose is to give a solution of this question. But we do not know the method which constructs the estimates for $N$ and for $p$ under such situation. The essential body of this question depends on the situation which we have only one category for data and have two parameters to be estimated. Hence in order to improve such situation we introduce a certain sequential procedure. Now we will let another inspector to examine independently the same lot. Then we may have sometime defectives pointed out by the first and the second inspectors which are divided into three categories as follows; two categories are respectively those of defectives pointed by the first inspector alone and by the second inspector alone and the last category is one of defectives pointed by both commonly. If the last does not empty, then we shall accomplish to improve the first situation, because we have three categories for data while the parameters to be estimated are three, that is, $N, p_{1}$, and $p_{2}$. However, if it is empty, then we shall not accomplish our aim, for we have only two categories for three parameters. Here-in we shall let the third inspector to examine the same lot and so on. In such way we shall introduce the following sequential procedure; 
(i) Let the inspectors continue one after another to examine the same lot independently, until it appears that at least two among them point out commonly the same defectives.

(ii) Stop the examination at the first $m$ when the $m$-th inspector points out the same defective commonly with at least one among $m-1$ inspectors.

(iii) Make the estimates for $N$ and $p_{1}, p_{2}, \ldots, p_{m}$, where $p_{i}(i=1,2, \ldots$ ,$m$ ) is a ratio of correct checks for defectives by the $i$-th inspector. [See Section 4]

Example. (a) Trial 1. The first inspector points out the items (1), (4.) and he overlooks the item (2). The second inspector points out the items (2), (4) and he overlooks the item (1). The item (4) is pointed by two inspectors and then the examination is stopped. By Remark 2 in Section 4 the estimates $N, p_{1}, p_{2}$ are respectively equal to $4,1 / 2,1 / 2$.

Examples of Examinations for Lot whose Size is 6 and which has 3 Defectives (unknown)

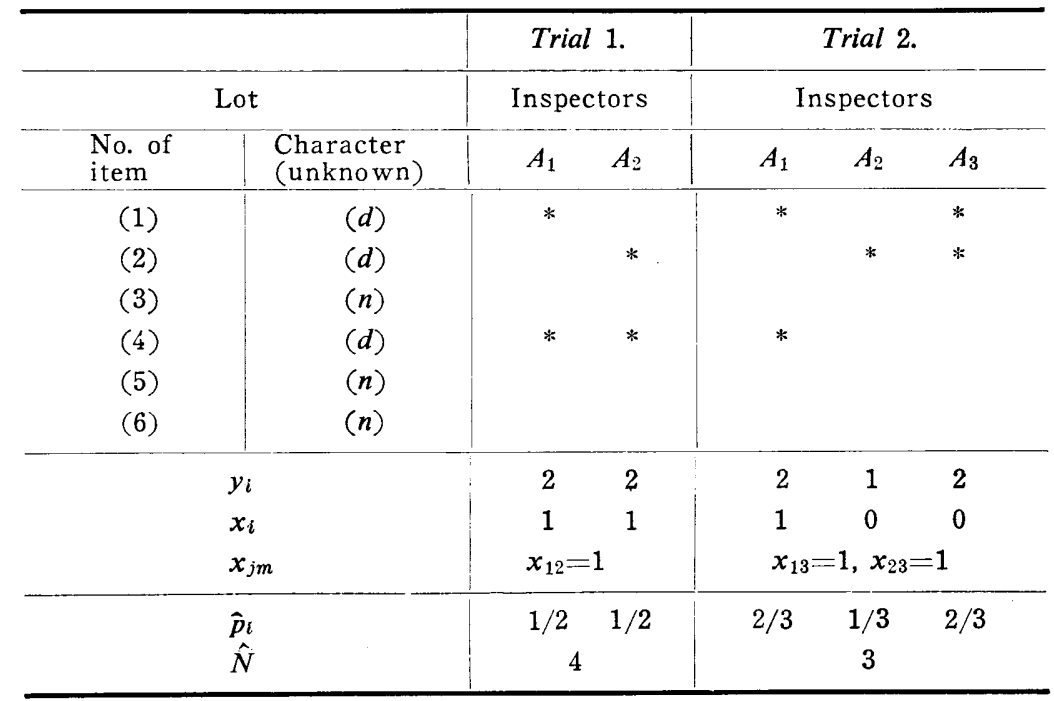

$(d)=$ defective, $(n)=$ non-defective. The mark $*$ denotes the event that the inspector on column points out a corresponding defective, while the no-mark defectives are overlooked by them. $y_{i}, x_{i}, x_{j}$ are defined in Section 2.

(b) Trial 2. The first inspector points out the items (1), (4) and the second inspector points out only the item (2). Hence the third inspector is put in and he points out the items (1), (2). Then the examination is stopped, for the item (1) and (2) are pointed commonly with the third inspector respectively by the first and by the second. By Remark 1 in Section 4 the estimates $\hat{N}, \hat{p}_{1}, \hat{p}_{2}$ and $\hat{p}_{3}$ are respectively equal to $3,2 / 3,1 / 3$, and $2 / 3$.

Section 2 defines certain notations and introduces some assumptions 
and discusses the stopping rule. The probability distributions of several random variables are shown in Section 3 and those of inspector numbers are tabulated for $N=1,2, \ldots, 10$, for $p_{i}=1 / 2$ and $3 / 4$ in Table 2 . In Section 4 we defines the estimates $\hat{N}, \hat{p}_{i}$ which are constructed by the method that is analogous to the maximum likelihood one. Finally the behavior of our procedure is discussed in Section 5 and by its discussion we may say that our procedure is available for $p_{i}>1 / 2$.

2. Notation and Assumption We shall consider a fixed lot of any size which contains $N$ defectives. The group of inspectors $A_{1}, A_{2}, \ldots$ are prepared for examinations of this lot. $E_{i}^{(j)}(i=1,2, \ldots ; j=1,2, \ldots, N)$ denotes an event that an inspector $A_{i}(i=1,2, \ldots)$ checks off the $j$-th defective $(j=1,2, \ldots, N)$ in the lot and $\bar{E}_{b}^{(j)}$ denotes the complement event of $E_{i}^{(i)}$. A probability of $E_{i}^{(j)}$ is denoted by $p_{i}^{(j)}$. Random variable $Y_{i}^{(j)}$ is defined such that its value is 1 when $E_{i}^{(j)}$ occurs and 0 when it does not occurs, and $Y_{i}$ denotes the sum $Y_{i}^{(1)}+Y_{i}^{(2)}+\ldots+Y_{i}^{(N)}$. Hence the event $\left\{Y_{i}\right.$ $\left.=y_{i}\right\}$ means that the inspector $A_{i}$ checks off $y_{i}$ defectives from the fixed lot and overlooks $N-y_{i}$ defectives. $X_{i m}$ is defined by $Y_{i}^{(1)} \cdot Y_{m}^{(1)}+Y_{i}^{(2)} \cdot Y_{m}^{(2)}+\ldots$ $+Y_{i}^{(N)} \cdot Y_{m}^{(, N)}$ and then $\left\{X_{i m}=x_{i m}\right\}$ means the event that both inspectors $A_{i}$ and $A_{m}$ check off commonly the same $x_{i m}$ defectives from the fixed lot. $X_{i}$ denotes the difference $Y_{i}-X_{i m}(i=1,2, \ldots, m-1)$ and $X_{m}=Y_{m}-\sum_{i=1}^{m-1} X_{i m}$, and then $X_{i}$ means the number which $A_{i}$ checks off alone from $N$ defectives in the lot.

Now $\mathfrak{J}$ denotes a class of every subsets consisted of $N$ integers $1,2, \ldots$, $N$ where each of those integers corresponds to each of defectives in the lot and then it contains $2^{N}$ subsets. Let the inspector $A_{1}$ pointed out $y_{1}$ defectives corresponding to $J_{1} \in \mathfrak{\Im}$ and $A_{2}$ pointed out $y_{2}$ defectives corresponding to $J_{2} \in \breve{J}$, and those event means that the event

$$
\left[\bigcap_{j \in J_{1}} E_{1}^{(j)} \cdot \bigcap_{j \in J_{1}} E_{1}^{(j)}\right] \frown\left[\bigcap_{j \in J_{2}} E_{2}^{(j)} \cdot \bigcap_{j \in J_{2}} \bar{E}_{2}^{(j)}\right]
$$

occurred where the sizes of set $J_{1}$ and $J_{2}$ are respectively $y_{1}$ and $y_{2}$. If $J_{1} \frown J_{2} \neq \phi$ where $\phi$ is empty set, then we stop toput the inspector into the lot and if $J_{1} \frown J_{2}=\phi$, then we invite the third inspector $A_{3}$ to examine the lot. Let $A_{3}$ checks off $y_{3}$ defectives corresponding to $J_{3} \in \mathfrak{\Im}$. If $\left[J_{1} \smile J_{2}\right]$ $\frown J_{3} \neq \phi$, then our operation is stopped and if $\left[J_{1} \smile J_{2}\right] \frown J_{3}=\phi$, then it is continued and so on. In such way our procedure defines the stopping rule as follows ;

(i) If $J_{1} \frown J_{2} \neq \phi$, then our operation is stopped and if $J_{1} \frown J_{2}=\phi$, then it is continued.

(ii) In general if we have $\left[J_{1} \smile J_{2} \smile \ldots \smile J_{m-1}\right] \frown J_{m} \neq \phi$ for some $m$ at first after $J_{1} \frown J_{2}=\phi,\left[J_{1} \smile J_{2}\right] \frown J_{3}=\phi, \ldots,\left[J_{1} \smile J_{2} \smile \ldots \smile J_{m-2}\right] \frown J_{m-1}=\phi$, then our operation is stopped at the $m$-th inspector. 
We define the new random variable $M$ as the first $m$ such that $\left[J_{1} \smile\right.$ $\left.J_{2} \smile \ldots \smile J_{m-1}\right] \frown J_{m} \neq \phi$ appears on our operation.

Finally we shall put the following assumptions;

(i) Events $E_{i}^{(1)}, E_{i}^{(2)}, \ldots, E_{i}^{(v)}$ are mutually independent for any $i$ and their probabilities are equal to $p_{i}$ for all defectives but may be dependent of the size of lot.

(ii) Sequences $\left\{E_{i}^{(1)}, E_{i}^{(2)}, \ldots, E_{i}^{(v)}\right\}(i=1,2, \ldots)$ are mutually independent.

(iii) The insqectors never make mistake for good items but the probabilites $p_{i}$ may be affected by their number which contributes to the size of lot.

\section{Probability Distributions of $\boldsymbol{M}$ and of $\left(\boldsymbol{X}_{i}, \boldsymbol{X}_{i m}, \boldsymbol{M}\right)$}

We shall require the probabilty that the event $\{M=\boldsymbol{m}\}$ occurs, that is, our operation stops at the $m$-th inspector. Let us consider the $j$-th defective. The probabilty that at least two among $k$ inspectors check off commonly the $j$-th defective is denoted by $P_{2, k}^{(j)}$. Then we have

$$
\begin{aligned}
1-P_{2, k}^{(j)} & =\operatorname{Pr} .\left\{\left[\bigcup_{i=1}^{k} E_{i}^{(j)} \cdot \bigcap_{h \neq i}^{k} \bar{E}_{h}^{(j)}\right] \smile\left[\bigcap_{i=1}^{k} \bar{E}_{i}^{(j)}\right]\right\} \\
& =\sum_{i=1}^{k} p_{i} \cdot \prod_{h \neq i}^{k}\left(1-p_{h}\right)+\prod_{i=1}^{k}\left(1-p_{i}\right),
\end{aligned}
$$

because of our assumption (i) and (ii). Multipling from 1 to $N$ for $j$ the events and using our assumption, we can observe that the probability that our operation does not stop at the $k$-th inspector is

$$
\operatorname{Pr} \text {. }\{\text { not stop at the } k \text {-th }\}=[(3.1)]^{N} \text {. }
$$

Therefore we get

Theorem 3.1. The probability that our operation stops at the $m$-th inspector is

$$
\begin{array}{r}
\operatorname{Pr} .\{M=2\}=1-\left(1-P_{2,2}\right)^{N}, \\
\operatorname{Pr} .\{M=m\}=\left(1-P_{2, m-1}\right)^{N}-\left(1-P_{2, m}\right)^{N} \\
\text { for } \quad m=3,4, \ldots
\end{array}
$$

where

$$
1-P_{2, k}=\sum_{i=1}^{k} p_{i} \prod_{h \neq i}^{k}\left(1-p_{h}\right)+\prod_{i=1}^{k}\left(1-p_{i}\right)
$$

Next, let the operation stop at the $m$-th inspector and we have observations $y_{1}=x_{1}+x_{1 m}, y_{2}=x_{2}+x_{2 m}, \ldots, y_{m-1}=x_{m-1}+x_{m-1, m}$ and $y_{m}=x_{m}+\sum_{j=1}^{m-1} x_{j m}$. Of course, $\sum_{j=1}^{m-1} x_{j m}$ is not zero, because it is the number of integers belonging to the set $\left[J_{1} \smile J_{2} \smile \ldots \smile J_{m-1}\right] \frown J_{m}$ which is not empty.

Theorem 3.2. The probability of the event $\left\{X_{i}=x_{i}, X_{j m}=x_{j m}, M=m\right.$; 
$1 \leq i \leq m, 1 \leq j \leq m-1\}$ is

$$
\begin{aligned}
p\left(x_{1}, x_{2}, \ldots, x_{m}, x_{1 m}, x_{2 m}, \ldots, x_{m-1 m}, m\right) \\
\quad=C(m) p_{m}^{z}\left(1-p_{m}\right)^{N-z} \prod_{i=1}^{m-1} p_{i}^{x_{i}+x_{i m}}\left(1-p_{i}\right)^{N-x_{i}-x_{i m}} .
\end{aligned}
$$

for $1 \leq z$ and $\sum_{i=1}^{m-1} x_{i}+z \leq N$, where $z=x_{m}+\sum_{j=1}^{m-1} x_{j m}$ and

$$
C(m)=\frac{N !}{\prod_{i=1}^{m} x_{i} ! \cdot \prod_{j=1}^{m-1} x_{j m} ! \cdot\left(N-\sum_{i=1}^{m} x_{i}-\sum_{j=1}^{m-1} x_{j m}\right) !} .
$$

Proof. Let sets $J_{1}, J_{2}, \ldots, J_{m}$ correspond to data. Then from our stopping rule any $j(1 \leq j \leq N)$ must belong to one of either two sets or sets of two types; two sets mean $J_{m}-J_{m} \frown\left[J_{1} \smile J_{2} \smile \ldots \smile J_{m-1}\right]$ and $\overline{J_{1} \smile J_{2} \smile \ldots \smile J_{m}}$ and two types mean such as $J_{i}-\left[J_{i} \frown J_{m}\right]$ and $J_{i} \frown J_{m}$. Then for a fixed $j$ we have the events and their probabilities as follows;

Set including $j$

$$
\begin{aligned}
& J_{m}-J_{m} \frown\left[J_{1} \smile J_{2} \smile \ldots \smile J_{m-1}\right]
\end{aligned}
$$

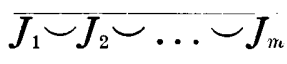

$$
\begin{aligned}
& J_{i}-\left[J_{i} \frown J_{m}\right] \\
& J_{i} \frown J_{m} \\
& (i=1,2, \ldots, m-1) \text {. }
\end{aligned}
$$

\section{Event}

$$
E_{m}^{(j)} \cdot \bigcap_{h=1}^{m-1} E_{h}^{(j)}
$$$$
\bigcap_{h=1}^{n} \overline{E_{h}^{(j)}}
$$$$
E_{i}^{(j)} \cdot \bigcap_{h \neq i}^{m} \overline{E_{h}^{(j)}}
$$$$
E_{i}^{(j)} \cdot E_{m}^{(j)} \cdot \prod_{h \neq i}^{m-1} \bar{E}_{h}^{(i)}
$$$$
p_{i} \cdot p_{m} \cdot \prod_{h \neq i}^{m-1}\left(1-p_{h}\right)
$$

Using the assumptions and that sizes of above sets take respectively $x_{m}$, $N-\sum x_{i}-\sum x_{j m}, x_{i}$ and $x_{i m}$, we get the probability for fixed $\left\{J_{1}, J_{2}, \ldots, J_{m}\right\}$, by multipling for $i$ from 1 to $m-1$ and for $j$ from 1 to $N$,

$$
\begin{aligned}
\prod_{i=1}^{m}\left[p_{i} \cdot \prod_{n \neq i}^{m}\left(1-p_{h}\right)\right]^{x_{i} i} \prod_{i=1}^{m-1}\left[p_{i} \cdot p_{m}\right. & \left.\cdot \prod_{h \neq i}^{m-1}\left(1-p_{h}\right)\right]^{x_{i m}} \\
& \times \prod_{i=1}^{m}\left(1-p_{i}\right)^{N-\Sigma x_{i}-\Sigma x_{j m}} .
\end{aligned}
$$

Now let us notice that the above probability depends only on sizes of sets constructed by sets $J_{1}, J_{2}, \ldots, J_{m}$. If such sizes are fixed, then the number of different classes becomes $C(m)$, of which the corresponding events are mutually exclussive, which proves Theorem 3.2.

4. Estimates of $\boldsymbol{N}$ and $\boldsymbol{p}_{i} \quad$ Let $\theta$ denote $\sum x_{i}+\sum x_{j m}$. $\theta$ means the size of the set $J_{1} \smile J_{2} \smile \ldots \smile J_{m}$, that is, whole number of defectives checked by $m$ inspectors, but not the sum of numbers checked. Then the estimate $\hat{N}$ of $N$ must satisfy the condition $N \geq \theta$. Let $f\left(p_{1}, p_{2}, \ldots, p_{m}, N\right)$ denote the likelihood function of (3.4). From the maximizing conditions for $N$ 


$$
\frac{f\left(p_{1}, p_{2}, \ldots, p_{m}, N\right)}{f\left(p_{1}, p_{2}, \ldots, p_{m}, N-1\right)} \geq 1 \text { and } \frac{f\left(p_{1}, p_{2}, \ldots, p_{m}, N\right)}{f\left(p_{1}, p_{2}, \ldots, p_{m}, N+1\right)} \geq 1
$$

we have the inequalities

$$
N \cdot \prod_{i=1}^{m}\left(1-p_{i}\right) \geq N-\theta
$$

and

$$
(N+1) \cdot \prod_{i=1}^{m}\left(1-p_{i}\right) \leq N+1-\theta .
$$

Differentiating $\log f$ by $p_{i}$ and putting $\frac{\partial \log f}{\partial p_{i}}=0$, we have equations

$$
p_{i}=\frac{y_{i}}{N}, \quad i=1,2, \ldots, m .
$$

Substituting (4.3) in (4.1) and (4.2), we obtain

$$
\prod_{i=1}^{m}\left(N-y_{i}\right) \geq N^{m-1} \cdot(N-\theta)
$$

and

$$
(N+1) \cdot \prod_{i=1}^{m}\left(N-y_{i}\right) \leq N^{m} \cdot(N+1-\theta) .
$$

These necessary conditions for $N$ do not uniquely determine our estimate $\hat{N}$. Nevertheless we propose to introduce, as an estimate for $N$, the largest integer $N$ satisfying the inequality (4.4). This estimate clearly satisfies the inequality (4.5). Moreover the following Theorem 5.3 and Theorem 5.4 will somewhat justify our proposition.

\section{Remark 1. The equation}

$$
N^{m-1} \cdot(N-\theta)-\prod_{i=1}^{m}\left(N-y_{i}\right)=0
$$

has one and only one root in the interval $N \geq \theta$. Therefore, we can easily determine an estimate $\hat{N}$.

This fact is proved as follows ; (i) first, if some $i$ exists such that $y_{i}$ is equal to $\theta$, then we have an unique solution $\hat{N}=\theta$ satisfying $\hat{N} \geq \theta$, and (ii) secondly we suppose $y_{i}<\theta$ for all $i$, and consider the function

$$
\varphi(N)=\log N^{m-1}(N-\theta)-\sum_{i=1}^{m} \log \left(N-y_{i}\right) .
$$

As $N$ increases from $\theta$ to $\infty$, the function $\varphi(N)$ starts from $-\infty$ and monotonously increases to its maximum, and hereafter decreases steadily to zero. The equation $\varphi(N)=0$ posesses only one root in the interval $N \geq \theta$.

Remark 2. In the case where $m=2$, we have $\hat{N}=\left[y_{1} y_{2} / x_{12}\right]$ for $N$ and for $p_{1}, p_{2}$ we get $\hat{p}_{1}=y_{1} / \hat{N}, \hat{p}_{2}=y_{2} / \hat{N}$. 
5. Behavior of procedure In this section we shall consider the behavior of our procedure. First we can see its real utility in the mean number of inspector and in the probability that it is less than $m$ inspectors. We can easily show the following theorem from (3.2).

Theorem 5.1. Let $E\{M\}$ denote the mean number of inspector, then

$$
E\{M\}=2+\sum_{k=2}^{\infty}\left(1-P_{2, k}\right)^{N}
$$

and let $P(m)$ denote the probability that inspectors less than $m$ are put in the lot, then

$$
P(m)=1-\left(1-P_{2, m}\right)^{N} \text {. }
$$

Corollary 5.1. Put $p_{i}=p(i=1,2, \ldots)$, then

$$
E\{M\}=2+\sum_{k=0}^{N}\left(\begin{array}{l}
N \\
k
\end{array}\right) p^{k} L_{k}(\beta, N),
$$

where

$$
L_{k}(\beta, N)=\beta \frac{d}{d \beta} L_{k-1}(\beta, N) \quad(k=1,2, \ldots),
$$

putting

$$
L_{0}(\beta, N)=\frac{\beta}{1-\beta} \text {, and } \beta=(1-p)^{N} .
$$

In practice, it is not far from real situations even if the ratios of correct checks are nearly equal to $1 / 2$. We mark at the points that $p_{i}$ are $1 / 2$ and 3/4. We prepared for small $N, E\{M\}$ and $P(m)$ in Table 1 and Table 2. Regarding to $N$ larger than $10, E\{M\}$ is nearly 2 and $P\{M=2\}$ is larger than $95 \%$. Therefore it may be useful to calculate the conditional expectation $E\{\hat{N}, M=2\}$ in Table 3 . The calculation of Table 3 are obtained by

$$
\begin{aligned}
& \left(1-a^{N}\right) E\{\hat{N} \mid M=2\} \\
& =N\left(1-a^{N}\right)-N\left(1-p_{1}\right)\left(1-p_{2}\right)\left(1-a^{N-1}\right) \\
& \quad+N(N-1) p_{1} p_{2}\left(1-p_{1}\right)\left(1-p_{2}\right) \cdot \sum_{k=1}^{N-2} \frac{\left(1-a^{k}\right) a^{N-2-k}}{k}
\end{aligned}
$$

where $a$ denotes $1-p_{1} p_{2}$. A simple calculation shows that

$$
\lim _{N \rightarrow \infty}(N-1) \sum_{k=1}^{N-2} \frac{\left(1-a^{k}\right) a^{N-2-k}}{k}=\frac{1}{1-a}=\frac{1}{p_{1} p_{2}} .
$$

Now we shall show the behavior of $\hat{N}$ for large $N$.

Lemma 1. The estimate $\hat{N}$ cannot exceed $N(N+1) / 2$.

Proof. It is clear that the estimate is equal to or differs by less than 1 from the greatest root of the equation

$$
Z^{m-1}(Z-\theta)-\prod_{i=1}^{m}\left(Z-y_{i}\right)=0 \text {. }
$$


Here we regard at the following facts. First the root of equation (5.5) is equal or less than that of equation

$$
Z^{m-1}(Z-N)-\left(Z-y_{j}-\theta^{\prime}\right) \prod_{i \neq j}^{m}\left(Z-y_{i}\right)=0,
$$

where (5.6) is obtained from (5.5) by putting $N=\theta+\theta^{\prime}$ in place of $\theta$ and $y_{j}^{\prime}=y_{j}+\theta^{\prime}$ in place of $y_{j}$. Therefore there always exists a root among the largest roots of (5.6) such that it is not less than that of (5.5) for fixed $\theta$. We have only to find the upper limit for the greatest root of (5.6). Secondly, in (5.6) the upper limit for the case $\sum_{j=1}^{m-1} x_{j m} \geq 2$ is less than or equal to that of the case $\sum_{j=1}^{m-1} x_{j m}=1$. Consequently we require the upper limit under the condition $\sum_{j=1}^{m-1} x_{j m}=1$ and $\sum_{i=1}^{m} y_{i}=N+1$. Such upper limit is obtained from the equation

$$
Z^{N}(Z-N)-(Z-1)^{N+1}=0,
$$

which is led by putting $m=N+1, y_{1}=y_{2}=\ldots=y_{N+1}=1$ and $\theta=N$ in (5.5). The left hand side of this equation is always positive in the interval $Z>N(N+1) / 2$. Hence we conclude that the greatest root of (5.5) does not exceed $N(N+1) / 2$.

Theorem 5.2. We have

$$
\begin{aligned}
\left(1-a^{N}\right) E\{\hat{N} / M=2\} & <E\{\hat{N}\} \\
& <\left(1-a^{N}\right) E\{\hat{N} / M=2\}+a^{N} \cdot \frac{N(N+1)}{2}
\end{aligned}
$$

and

$$
\lim _{N \rightarrow \infty} \frac{E\{\hat{N}\}}{N}=1 .
$$

Proof. The inequality (5.8) is immediate from

$$
E\{\hat{N}\}=\left(1-a^{N}\right) E\{\hat{N} / M=2\}+a^{N} \cdot E\{\hat{N} / M>2\} .
$$

It is easily shown that

$$
\lim _{N \rightarrow \infty} \frac{E\{\hat{N} / M=2\}}{N}=1
$$

from (5.3) and (5.4). Combining this fact and (5.8) in Theorem 5.2, we have the result in (5.9).

Next, we shall consider the mean square of error and the variance of estimate $\hat{N}$. We have

$$
E\left\{\hat{N}^{2}\right\}=\left(1-a^{N}\right) E\left\{\hat{N}^{2} / M=2\right\}+a^{N} \cdot E\left\{\hat{N}^{2} / M>2\right\} .
$$


Applying Lemma 1, we obtain the inequality

$$
\begin{aligned}
& \left(1-a^{v}\right) E\left\{\hat{N}^{2} / M=2\right\} \\
& \quad<E\left\{\hat{N}^{2}\right\}<\left(1-a^{v}\right) E\left\{N^{2} / M=2\right\}+\left\{\frac{N(N+1)}{2}\right\}^{2} \cdot a^{v},
\end{aligned}
$$

where $a=1-p_{1} p_{2}$. Further after a somewhat tedious calculation similar in method to that used for obtaining (5.3), we get the following result.

$$
\begin{aligned}
& \left(1-a^{v}\right) E\left\{\hat{N}^{2} / M=2\right\} \\
& =N p_{1} p_{2}+N(N-1) p_{1}^{2} p_{2}^{2}+2 N(N-1) p_{1} p_{2}\left\{p_{1}\left(1-p_{2}\right)+p_{2}\left(1-p_{1}\right)\right\} \\
& +4 N(N-1)\left(1-a^{N-2}\right) p_{1} p_{2}\left(1-p_{1}\right)\left(1-p_{2}\right) \\
& +N\left(1-a^{N-1}\right)\left\{p_{1}\left(1-p_{2}\right)+p_{2}\left(1-p_{1}\right)\right\} \\
& +N(N+1)\left(1-a^{N-2}\right)\left\{p_{1}^{2}\left(1-p_{2}\right)^{2}+p_{2}^{2}\left(1-p_{1}\right)^{2}\right\} \\
& +4 N(N-1) p_{1} p_{2}\left(1-p_{1}\right)\left(1-p_{2}\right) \cdot \sum_{k=1}^{N-2} \frac{a^{v-k-2}\left(1-a^{k}\right)}{k} \\
& +2 N(N-1)(N-2) p_{1} p_{2}\left(1-p_{1}\right)\left(1-p_{2}\right) \\
& \times\left\{p_{1}\left(1-p_{2}\right)+p_{2}\left(1-p_{1}\right)\right\} \cdot \sum_{k=1}^{N-3} \frac{a^{N-k-3}\left(1-a^{k}\right)}{k} \\
& +N(N-1) p_{1} p_{2}\left(1-p_{1}\right)\left(1-p_{2}\right) \\
& \times \sum_{k=1}^{N-2} \frac{1}{k}\left(\frac{1}{k}+\frac{1}{k+1}+\ldots+\frac{1}{N-2}\right) a^{N-k-2} \cdot\left(1-a^{k}\right) \\
& +N(N-1)(N-2) p_{1} p_{2}\left(1-p_{1}\right)\left(1-p_{2}\right)\left\{p_{1}\left(1-p_{2}\right)+p_{2}\left(1-p_{1}\right)\right\} \\
& \times \sum_{k=1}^{N-3} \frac{1}{k}\left(\frac{1}{k}+\frac{1}{k+1}+\ldots+\frac{1}{N-3}\right) a^{N-k-3} \cdot\left(1-a^{k}\right) \\
& +N(N-1)(N-2)(N-3) p_{1}^{2} p_{2}^{2}\left(1-p_{1}\right)^{2}\left(1-p_{2}\right)^{2} \\
& \times \sum_{k=1}^{N-4} \frac{1}{k}\left(\frac{1}{k}+\frac{1}{k+1}+\ldots+\frac{1}{N-4}\right) a^{N-k-4} \cdot\left(1-a^{k}\right) .
\end{aligned}
$$

By virtue of the limit theorem;

$$
\lim _{N \rightarrow \infty} N^{2} \cdot \sum_{k=1}^{N} \frac{1}{k}\left(\frac{1}{k}+\frac{1}{k+1}+\ldots+\frac{1}{N^{-}}\right) a^{N-k} \cdot\left(1-a^{k}\right)=\frac{1}{p_{1}^{2} p_{2}^{2}},
$$

we get the following result

$$
\lim _{N \rightarrow \infty} \frac{1}{N^{2}} E\left\{\hat{N}^{2} / M=2\right\}==1,
$$

which gives by virtue of (5.12)

$$
\lim _{N \rightarrow \infty} \frac{1}{N^{2}} E\left\{\hat{N}^{2}\right\}=1 .
$$


On the other hand we have as the mean square and variance of $\hat{N}$

$$
M(\hat{N})=E\left\{\hat{N}^{2}\right\}-2 E\{\hat{N}\} \cdot N+N^{2}
$$

and

$$
V(\hat{N})=E\left\{\hat{N}^{2}\right\}-[E\{\hat{N}\}]^{2} .
$$

By above results we can easily show the following theorem.

Theorem 5.3.

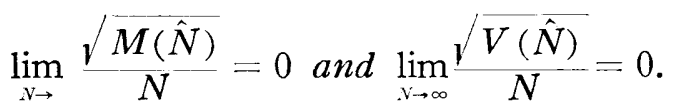

Remark 3. Theorem 5.2 does not mean the consistency of estimate although it is likely for one.

The exact probability distribution of $\hat{N}$ for small $N$ is found easily, but for $N$ larger than 4 it is difficult and needs heavy labour. In such circumstance we find the exact values of $E\{\hat{N}\}, V(\hat{N})$ and $M(\hat{N})$ for $N=1,2,3$. Their values are shown with brackets in Table 3 and Table 4.

We are thankful to our friend A. Ono for his advice about the convergence of (5.15).

\begin{tabular}{|c|c|}
\hline Case 2. & $p_{i}=3 / 4$ \\
\hline$N$ & $E\{M\}$ \\
\hline 1 & 2.6667 \\
\hline 2 & 2.2187 \\
\hline 3 & 2.0877 \\
\hline 4 & 2.0372 \\
\hline 5 & 2.0208 \\
\hline 6 & 2.0098 \\
\hline 7 & 2.0031 \\
\hline 8 & 2.0011 \\
\hline 9 & 2.0006 \\
\hline 10 & 2.0002 \\
\hline
\end{tabular}

TABLE 1. Expected Number $E\{M\}$ of Inspectors.

\begin{tabular}{cc}
\hline Case 1. & $p_{i}=1 / 2$ \\
\hline$N$ & $E\{M\}$ \\
\hline 1 & 4.000 \\
2 & 2.963 \\
3 & 2.584 \\
4 & 2.389 \\
5 & 2.272 \\
6 & 2.195 \\
7 & 2142 \\
8 & 2.102 \\
9 & 2.068 \\
10 & 2.057 \\
\hline
\end{tabular}

TABLE 2. Probability $p(m)$ that at most $m$ Inspectors are led. Case 1. $p_{i}=1 / 2$

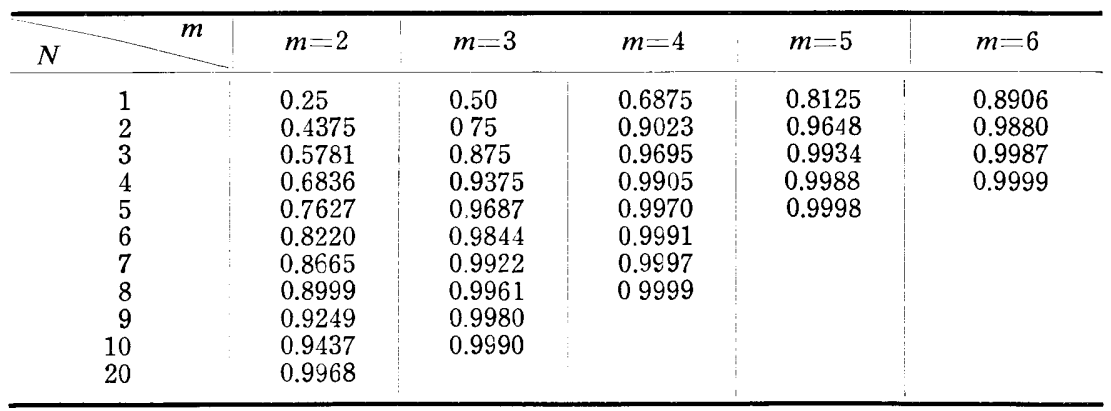


Estimate of the Number of Defectives when Inspectors may make Miss Checks

Case 2. $p_{i}=3 / 4$

\begin{tabular}{r|r|r|rr|c}
\hline \multicolumn{1}{|c|}{$m$} & $m=2$ & $m=3$ & $m=4$ & $m=5$ & $m=6$ \\
\hline 1 & 0.5625 & 0.8437 & 0.9492 & 0.9844 & 0.9954 \\
2 & 0.8086 & 0.9756 & 0.9974 & 0.9998 & \\
3 & 0.9163 & 0.9962 & 0.9999 & & \\
4 & 0.9634 & 0.9994 & & & \\
5 & 0.9840 & 0.9999 & & & \\
6 & 0.9930 & & & & \\
7 & 0.9969 & & & & \\
8 & 0.9987 & & & & \\
9 & 0.9994 & & & & \\
10 & 0.9997 & & & & \\
\hline
\end{tabular}

TABLE 3. Conditional Expectation $E\left\{\hat{N} \mid x_{12} \neq 0\right\}$ with Upper and Lower Bounds of $E\{\hat{N}\}$.

Case 1. $p_{i}=1 / 2$

\begin{tabular}{|c|c|c|c|}
\hline$N$ & $E\left\{\hat{N}: x_{12} \neq 0\right\}$ & $\begin{array}{l}\text { Lower Bound } \\
\quad \text { of } E\{\hat{N}\}\end{array}$ & $\begin{array}{l}\text { Upper Bound } \\
\text { of } E\{\hat{N}\}\end{array}$ \\
\hline $\begin{array}{l}1 \\
2 \\
3\end{array}$ & $\begin{array}{l}1.0000 \\
1.7143 \\
2.5946\end{array}$ & \multicolumn{2}{|c|}{$\left.\begin{array}{l}1.000 \\
1.778 \\
2.744\end{array}\right\}$} \\
\hline $\begin{array}{r}4 \\
5 \\
6 \\
7 \\
8 \\
9 \\
10 \\
11 \\
12\end{array}$ & $\begin{array}{r}3.6000 \\
4.6948 \\
5.8490 \\
7.0378 \\
8.2420 \\
9.4470 \\
10.6424 \\
11.8214 \\
12.9800\end{array}$ & $\begin{array}{r}2.461 \\
3.581 \\
4.808 \\
6.098 \\
7.417 \\
8.738 \\
10.043 \\
11.322 \\
12569\end{array}$ & $\begin{array}{r}5.625 \\
7.140 \\
8.546 \\
9.836 \\
11.021 \\
12.116 \\
13.140 \\
14.110 \\
15.040\end{array}$ \\
\hline
\end{tabular}

Case 2. $p_{i}=3 / 4$

\begin{tabular}{|c|c|c|c|}
\hline$N$ & $E\left\{\hat{N} \mid x_{12} \neq 0\right\}$ & $\begin{array}{l}\text { Lower Bound } \\
\quad \text { of } E\{\hat{N}\}\end{array}$ & $\begin{array}{c}\text { Upper Bound } \\
\text { of } E\{\hat{N}\}\end{array}$ \\
\hline $\begin{array}{l}1 \\
2 \\
3\end{array}$ & $\begin{array}{l}1.0000 \\
1.9130 \\
2.9640\end{array}$ & \multicolumn{2}{|c|}{$\left.\begin{array}{l}1.000 \\
1.920 \\
2.973\end{array}\right\}$} \\
\hline $\begin{array}{r}4 \\
5 \\
6 \\
7 \\
8 \\
9 \\
10 \\
11 \\
12\end{array}$ & $\begin{array}{r}4.0470 \\
5.1156 \\
6.1584 \\
7.1790 \\
8.1851 \\
9.1832 \\
10.1777 \\
11.1712 \\
12.1647\end{array}$ & $\begin{array}{r}3.899 \\
5.034 \\
6.115 \\
7.157 \\
8.174 \\
9.178 \\
10.175 \\
11.170 \\
12.164\end{array}$ & $\begin{array}{r}4.265 \\
5.274 \\
6.262 \\
7.243 \\
8.222 \\
9.204 \\
10.189 \\
11.177 \\
12.168\end{array}$ \\
\hline
\end{tabular}


TABLE 4. Variance and Mean Square Error of $\hat{N}$ (their exact values for $N=1,2,3$ )

Case 1. $p_{i}=1 / 2$

\begin{tabular}{r|c|c}
\hline$N$ & $V\left[\hat{N} \mid x_{12} \neq 0\right]$ & $M\left[\hat{N} x_{12} \neq 0\right]$ \\
\hline 1 & 0.000 & 0.000 \\
2 & 0.204 & 0.286 \\
& $(0.173)$ & $(0.222)$ \\
3 & 0.728 & 0.892 \\
& $(0.778)$ & $(0.843)$ \\
4 & 1.584 & 1.744 \\
5 & 2.809 & 2.902 \\
6 & 4.457 & 4.480 \\
7 & 6.578 & 6.579 \\
8 & 9.190 & 9.249 \\
9 & 12.257 & 12.456 \\
1.0 & 15.760 & 16.1 .73 \\
11 & 19.615 & 20.290 \\
12 & 23.723 & 24.683 \\
\hline
\end{tabular}

Case 2. $p_{i}=3 / 4$

\begin{tabular}{r|c|c}
\hline$N$ & $V\left[\hat{N} \mid x_{12} \neq 0\right]$ & $M\left[\hat{N} \mid x_{12} \neq 0\right]$ \\
\hline 1 & 0.000 & 0.000 \\
2 & 0.080 & 0.087 \\
& $(0.074)$ & $(0.080)$ \\
3 & 0.308 & 0.310 \\
& $(0.305)$ & $(0.306)$ \\
4 & 0.649 & 0.651 \\
5 & 1.055 & 1.068 \\
6 & 1.454 & 1.479 \\
7 & 1.789 & 1.821 \\
8 & 2.031 & 2.065 \\
9 & 2.187 & 2.221 \\
10 & 2.249 & 2.280 \\
11 & 2.333 & 2.363 \\
12 & 2.373 & 2.400 \\
\hline
\end{tabular}

